\title{
A first determination of parton distributions with theoretical uncertainties
}

\author{
Rabah Abdul Khalek ${ }^{1,2}$, Richard D. Ball ${ }^{3}$, Stefano Carrazza ${ }^{4}$, Stefano Forte ${ }^{4, a}{ }_{\mathbb{C}}$, Tommaso Giani ${ }^{3}$, \\ Zahari Kassabov ${ }^{5}$, Emanuele R. Nocera ${ }^{2}$, Rosalyn L. Pearson ${ }^{3}$, Juan Rojo ${ }^{1,2}$, Luca Rottoli ${ }^{6,7}$, \\ Maria Ubiali $^{8}$, Cameron Voisey $^{5}$, Michael Wilson $^{3}$ \\ ${ }^{1}$ Department of Physics and Astronomy, VU Amsterdam, De Boelelaan 1081, 1081, HV, Amsterdam, The Netherlands \\ 2 Nikhef, Science Park 105, 1098, XG Amsterdam, The Netherlands \\ ${ }^{3}$ The Higgs Centre for Theoretical Physics, University of Edinburgh, JCMB, KB, Mayfield Rd, Edinburgh EH9 3JZ, Scotland \\ 4 Tif Lab, Dipartimento di Fisica, Università di Milano and INFN, Sezione di Milano, Via Celoria 16, 20133 Milan, Italy \\ ${ }^{5}$ Cavendish Laboratory, University of Cambridge, Cambridge CB3 OHE, UK \\ ${ }^{6}$ Dipartimento di Fisica G. Occhialini, U2, Università degli Studi di Milano-Bicocca, Piazza della Scienza, 3, 20126 Milan, Italy \\ ${ }^{7}$ INFN, Sezione di Milano-Bicocca, 20126 Milan, Italy \\ ${ }^{8}$ DAMTP, University of Cambridge, Wilberforce Road, Cambridge CB3 0WA, UK
}

Received: 27 August 2019 / Accepted: 1 October 2019 / Published online: 10 October 2019

(C) The Author(s) 2019

\begin{abstract}
The parton distribution functions (PDFs) which characterize the structure of the proton are currently one of the dominant sources of uncertainty in the predictions for most processes measured at the Large Hadron Collider (LHC). Here we present the first extraction of the proton PDFs that accounts for the missing higher order uncertainty (MHOU) in the fixed-order QCD calculations used in PDF determinations. We demonstrate that the MHOU can be included as a contribution to the covariance matrix used for the PDF fit, and then introduce prescriptions for the computation of this covariance matrix using scale variations. We validate our results at next-to-leading order (NLO) by comparison to the known next order (NNLO) corrections. We then construct variants of the NNPDF3.1 NLO PDF set that include the effect of the MHOU, and assess their impact on the central values and uncertainties of the resulting PDFs.
\end{abstract}

The search for new physics at present [1] and future [2] highenergy colliders, and specifically at the LHC, has turned from the mapping of the energy frontier to the exploration of the precision frontier: looking for subtle deviations from Standard Model predictions. In this endeavor, an accurate estimate of uncertainties associated with these predictions is crucial. At present, these uncertainties have two main origins. The first is the missing higher order uncertainty (MHOU) from the truncation of the QCD perturbative expansion. The second is related to knowledge of the structure of the colliding protons, as encoded in the parton distributions (PDFs) [3].

a e-mail: stefano.forte@mi.infn.it
PDFs are extracted by comparing theoretical predictions to experimental data. Currently, PDF uncertainties only account for the propagated statistical and systematic errors on the measurements used in their determination. However, the same MHOU which affects predictions at the LHC also affect predictions for the various processes that enter the PDF determination. These are currently neglected, perhaps because they are believed to be generally less important than experimental uncertainties. However, as PDFs become more precise, in particular thanks to ever tighter constraints from LHC data [4], MHOUs in PDF determinations will eventually become significant. Already in recent PDF sets making extensive use of LHC data, such as NNPDF3.1 [5], the shift between PDFs at next-to-leading order (NLO) and the next order (NNLO) is sometimes larger than the PDF uncertainties from the experimental data.

Here we present the first PDF extraction that systematically accounts for the MHOU in the QCD calculations used to extract them. MHOUs are routinely estimated by varying the arbitrary renormalization $\mu_{r}$ and factorization $\mu_{f}$ scales of perturbative computations [1], though alternative methods have also been proposed [6-8]. Our inclusion of the MHOU in a PDF fit involves two steps: first we establish how theoretical uncertainties can be included in such a fit through a covariance matrix $[9,10]$, and then we find a way of computing and validating the covariance matrix associated with the MHOU using scale variations [11]. By producing variants of NNPDF3.1 which include the MHOU, we are then able to finally address the long-standing question of their impact on state-of-the-art PDF sets. A detailed discussion of our results 
is presented in a companion paper [12], to which we refer for full computational details, definitions, proofs and results.

Assuming that theory uncertainties can be modeled as Gaussian distributions, in the same way as experimental systematics, then the associated theory covariance matrix $S_{i j}$ can be expressed in terms of nuisance parameters

$S_{i j}=\frac{1}{N} \sum_{k} \Delta_{i}^{(k)} \Delta_{j}^{(k)}$

where $\Delta_{i}^{(k)}=T_{i}^{(k)}-T_{i}^{(0)}$ is the expected shift with respect to the central theory prediction for the $i$-th cross-section, $T_{i}^{(0)}$, due to the theory uncertainty, and $N$ is a normalization factor determined by the number of independent nuisance parameters. Since theory uncertainties are independent of the experimental ones, the two can be combined in quadrature: the $\chi^{2}$ used to assess the agreement of theory and data is given by

$\chi^{2}=\sum_{i, j=1}^{N_{\mathrm{dat}}}\left(D_{i}-T_{i}^{(0)}\right)(S+C)_{i j}^{-1}\left(D_{j}-T_{j}^{(0)}\right)$,

with $D_{i}$ the central experimental value of the $i$-th datapoint, and $C_{i j}$ the experimental covariance matrix. More details of the implementation of the theory covariance matrix in PDF fits may be found in Refs. $[9,10]$.

The choice of nuisance parameters $\Delta_{i}^{(k)}$ used in Eq. (1) to estimate a particular theoretical uncertainty is not unique, reflecting the fact that such estimates always have some degree of arbitrariness. Here we focus on the MHOU, and choose to use scale variations to estimate $\Delta_{i}^{(k)}$. A standard procedure [1] is the so-called 7-point prescription, in which the MHOU is estimated from the envelope of results obtained with the following scales

$\left(k_{f}, k_{r}\right) \in\left\{(1,1),(2,2),\left(\frac{1}{2}, \frac{1}{2}\right),(2,1),(1,2),\left(\frac{1}{2}, 1\right),\left(1, \frac{1}{2}\right)\right\}$

where $k_{r}=\mu_{r} / \mu_{r}^{(0)}$ and $k_{f}=\mu_{f} / \mu_{f}^{(0)}$ are the ratios of the renormalization and factorization scales to their central values. Varying $\mu_{r}$ estimates the MHOU in the hard coefficient function of the specific process, while the $\mu_{f}$ variation estimates the MHOU in PDF evolution.

In order to compute a covariance matrix, we must not only choose a set of scale variations, but also make some assumptions about the way they are correlated. We do this by, first of all, classifying the input datasets used in PDF fits into processes as indicated in Table 1: charged-current (CC) and neutral-current (NC) deep-inelastic scattering (DIS), Drell-Yan (DY) production of gauge bosons (invariant mass, transverse momentum, and rapidity distributions), single-jet inclusive and top pair production cross-sections. Note that this step requires making an educated guess as to which crosssections are likely to have a similar structure of higher-order corrections.
Table 1 Classification of datasets into process types

\begin{tabular}{ll}
\hline Process type & Datasets \\
\hline DIS NC & NMC, SLAC, BCDMS, HERA NC \\
DIS CC & NuTeV, CHORUS, HERA CC \\
DY & CDF, D0, ATLAS, CMS, LHCb $\left(y, p_{T}, M_{l l}\right)$ \\
JET & ATLAS, CMS inclusive jets \\
TOP & ATLAS, CMS total + differential cross-sections \\
\hline
\end{tabular}

Next, we formulate a variety of prescriptions for how to construct Eq. (1) by picking a set of scale variations and correlation patterns. A simple possibility is the 3-point prescription, in which we vary both scales coherently (thus setting $k_{f}=k_{r}$ ) by a fixed amount about the central value, independently for each process. More sophisticated prescriptions vary the two scales independently, but by the same amount, and assume that while $\mu_{r}$ is only correlated within a given process, $\mu_{f}$ is fully correlated among processes. This assumption is based on the observation that $\mu_{f}$ variations estimate the MHOU in the evolution equations, which are universal (process-independent), though it is an approximation given that the evolution of different PDFs is governed by different anomalous dimensions, which do not necessarily share the same MHO corrections.

We then proceed to the validation of the resulting covariance matrices at NLO. We use the same experimental data and theory calculations as in the NNPDF3.1 $\alpha_{s}$ study [13] with two minor differences: the value of the lower kinematic cut has been increased from $Q_{\min }^{2}=2.69 \mathrm{GeV}^{2}$ to $13.96 \mathrm{GeV}^{2}$ in order to ensure the validity of the perturbative QCD expansion when scales are varied downwards, and the HERA $F_{2}^{b}$ and fixed-target Drell-Yan cross-sections have been removed, for technical reasons related to difficulties in implementing scale variation. In total we then have $N_{\text {dat }}=2819$ data points. The theory covariance matrix $S_{i j}$ has been constructed by means of the ReportEng ine software [14] taking as input the scale-varied NLO theory cross-sections $T_{i}\left(k_{f}, k_{r}\right)$, provided by APFEL [15] for the DIS structure functions and by APFELgrid [16] combined with APPLgrid [17] for the hadronic cross-sections.

Since for the processes in Table 1 the NNLO predictions are known, we can validate the NLO covariance matrix against the known NNLO results. For this exercise, a common input NLO PDF is used in both cases. In order to validate the diagonal elements of $S_{i j}$, which correspond to the overall size of the MHOU, we first normalize it to the central theory prediction, $\widehat{S}_{i j}=S_{i j} / T_{i}^{(0)} T_{j}^{(0)}$. Then we compare in Fig. 1 the relative uncertainties, $\sigma_{i}=\sqrt{\widehat{S}_{i i}}$ to the relative shifts between predictions at NLO and NNLO, $\delta_{i}=$ $\left(T_{i}^{(0) \text {,nnlo }}-T_{i}^{(0), \text { nlo }}\right) / T_{i}^{(0) \text {,nlo }}$, for each of the $N_{\text {dat }}=2819$ observables. In all cases, $\delta_{i}$ turns out to be smaller or com- 


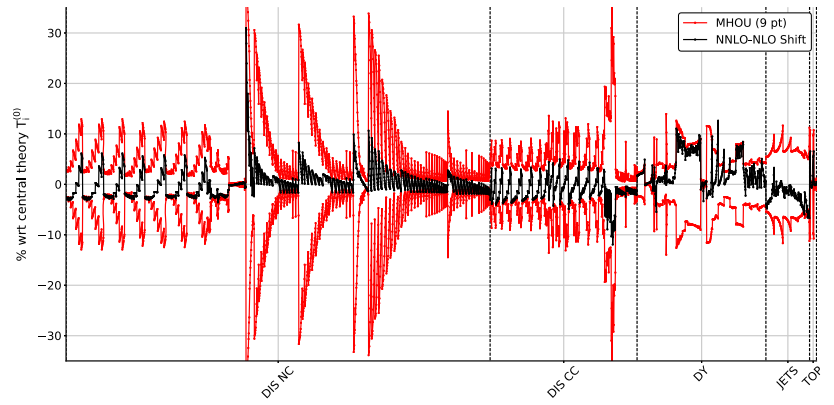

Fig. 1 The relative uncertainties $\sigma_{i}$ (9-point prescription) on the 2819 datapoints used in the PDF fit, compared to the known NLO-NNLO relative shifts $\delta_{i}$ in theory prediction

parable to $\sigma_{i}$, showing that this prescription provides a good (if somewhat conservative) estimate of the diagonal theory uncertainties.

The validation of the full covariance matrix including correlations is more subtle. We first diagonalize $\widehat{S}_{i j}$, by finding the (orthonormal) eigenvectors $e_{i}^{a}$ which correspond to positive eigenvalues $\left(s^{a}\right)^{2}$ : these define a subspace $S$ orthonormal to the large null subspace. The dimension $N_{S}$ of $S$ depends on the total number of independent scale variations, the number of processes, and the correlation pattern. Its determination is nontrivial, and it requires computing firstly the total number of distinct scale variations for any pair of processes, i.e., the total number of vectors $\Delta^{(k)}$ in Eq. (1), and secondly determining the full set of linear relations between them in order to establish how many of them are independent (see Ref. [12]).

For the 5 processes in Table 1, and the 9-point prescription, we find $N_{S}=28$, while for the simpler 3-point prescription $N_{S}=6$. We then compute the $N_{S}$ projections $\delta^{a}$ of the NLONNLO shifts $\delta_{i}$ along each eigenvector, and compare them to the square root of the corresponding eigenvalues, $s^{a}$. Finally we compute the length $\left|\delta_{i}^{\text {miss }}\right|$ of the remaining component of the vector $\delta_{i}$ that lies in the null subspace of $\widehat{S}$.

The validation can be considered successful if the angle $\theta=\arcsin \left(\left|\delta_{i}^{\mathrm{miss}}\right| /\left|\delta_{i}\right|\right)$ is small, meaning that the NNLONLO shift lies substantially within the subspace $S$ estimated by the scale variations, and furthermore if $\left|\delta^{a}\right| \simeq\left|s^{a}\right|$, so that the size of the shift along each eigenvector is correctly estimated by the corresponding eigenvalue. Using the 9-point prescription, for individual processes we find $\theta=3^{\circ}, 14^{\circ}, 22^{\circ}, 32^{\circ}, 16^{\circ}$ for top, jets, DY, NC and CC DIS respectively. For the complete dataset with the same prescription we find $\theta=26^{\circ}$.

The projected shifts and eigenvalues are compared in Fig. 2. The size of the eigenvalues generally falls as the projected shifts get smaller. For the six largest eigenvectors the eigenvalue is always larger than the shift and, in all but two cases, of very similar size to the shift. The seventh eigenvalue is smaller than, but of the same order as, the shift, while the eighth eigenvalue significantly underestimates the
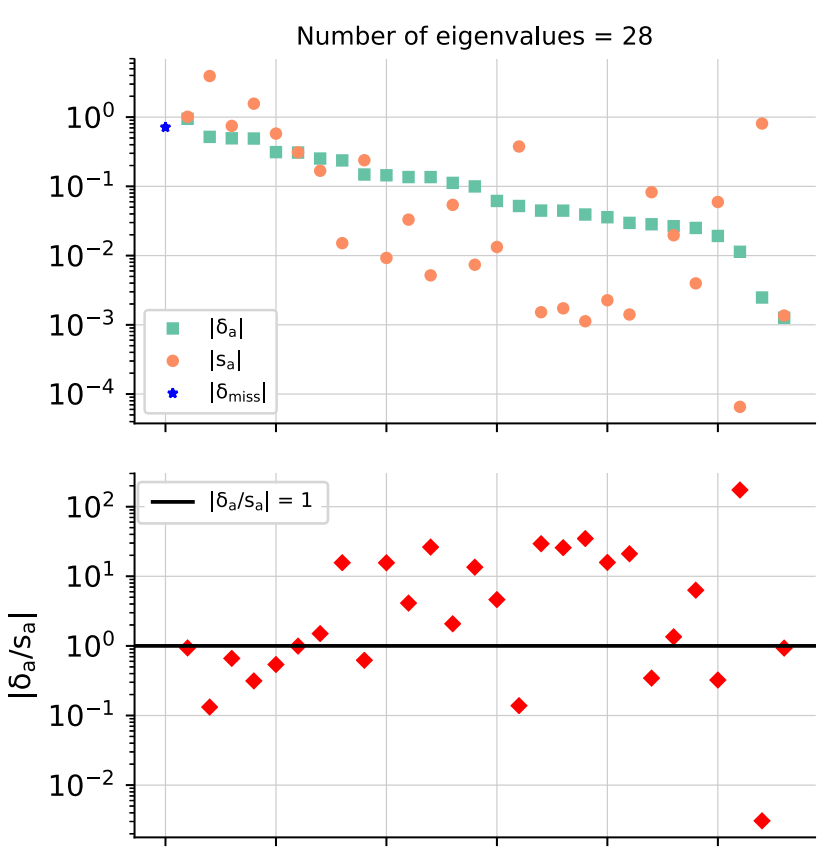

Fig. 2 The square root eigenvalues $s^{a}$ of the theory covariance matrix $\widehat{S}_{i j}$ computed using the 9-point prescription, and the projections $\delta^{a}$ of the NNLO-NLO shift vector $\delta_{i}$ on the eigenvectors. The length $\left|\delta_{i}^{\text {miss }}\right|$ of the component of $\delta_{i}$ lying in the null subspace of $\widehat{S}_{i j}$ is also shown

shift. However, given that the eighth eigenvalue is already one order of magnitude smaller that the first, this means that most of the shift is well described by the theory covariance matrix, and somewhat overestimated by it in just a few cases. We conclude that the validation is successful: remarkably, the pattern of correlations of theory shifts in a 2819-dimensional vector space is well captured by just 28 nuisance parameters.

Adding the theory covariance matrix $S_{i j}$ to the experimental covariance matrix $C_{i j}$, while increasing the diagonal uncertainty on each individual prediction, also (and perhaps more importantly) introduces a set of theory-induced correlations between different experiments and processes, even when the experimental data points are uncorrelated. This is illustrated in Fig. 3, showing the combined experimental and theoretical (9-point) correlation matrix: it is clear that sizable correlations appear even between experimentally unrelated measurements.

We can now proceed to a NLO global PDF determination with a theory covariance matrix $S_{i j}$ computed using the 9point prescription. From the point of view of the NNPDF fitting methodology, the addition of the theory contribution to the covariance matrix does not entail any changes: we follow the procedure of Ref. [18], but with the covariance matrix $C_{i j}$ now replaced by $C_{i j}+S_{i j}$, both in the Monte Carlo replica generation and in the fitting. In Table 2 we show some fit quality estimators for the resulting PDF sets obtained using only the experimental covariance matrix, alongside the theory covariance matrix with two different prescriptions. 
Experimental + Theory Correlation Matrix (9 pt)

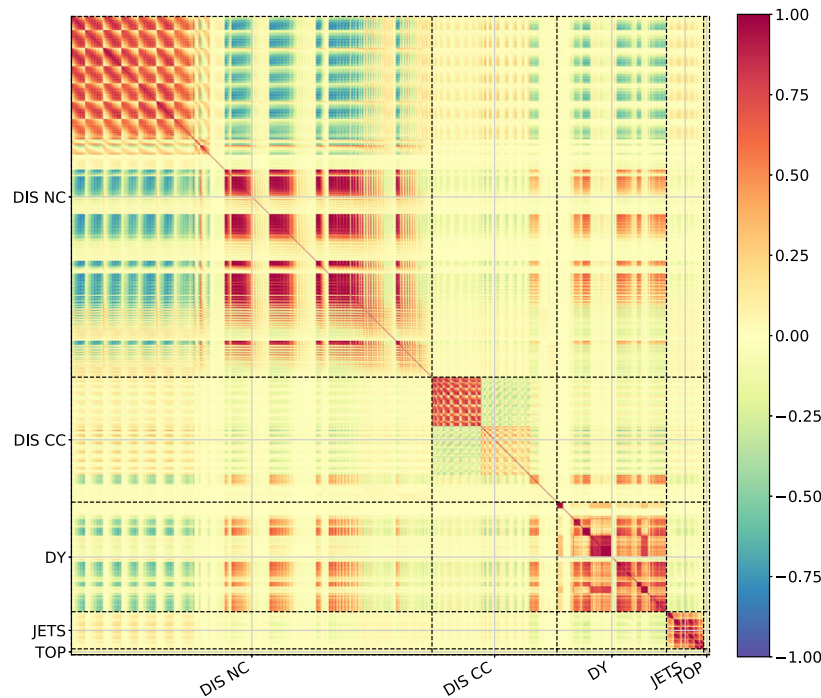

Fig. 3 The combined experimental and theoretical (9-point) correlation matrix for the $N_{\text {dat }}$ cross-sections in the fit

Table 2 The central $\chi^{2}$ per datapoint and the average uncertainty reduction $\phi$ for the 3-point and 9-point fits

\begin{tabular}{llll}
\hline & $C$ & $C+S^{(3 \mathrm{pt})}$ & $C+S^{(9 \mathrm{pt})}$ \\
\hline$\chi^{2}$ & 1.139 & 1.139 & 1.109 \\
$\phi$ & 0.314 & 0.394 & 0.415
\end{tabular}

In particular, we show the $\chi^{2}$ per datapoint and the $\phi$ estimator [18], which gives the ratio of the uncertainty in the predictions using the output PDFs to that of the original data, averaged in quadrature over all data. The quality of the fit is improved by the inclusion of the MHOU, with the 9-point prescription performing rather better than 3-point. Interestingly, $\phi$ only increases by around 30\% when one includes the theory covariance matrix, much less than the $70 \%$ one would expect taking into account the relative size of the NLO MHOU and experimental uncertainties. This means that in the region of the data, taking the MHOU into account increases the PDF uncertainties only rather moderately. This suggests that the addition of the MHOU is resolving some of the tension between data and theory, so that the larger overall uncertainty is partly compensated by the improved fit quality, though of course the highly correlated nature of theory uncertainties also plays a role in reducing their impact.

In Fig. 4 we compare at $Q=10 \mathrm{GeV}$ the gluon and quark singlet PDFs obtained at NLO with and without a theory covariance matrix, normalized to the latter. We also show the central NNLO result when the theory covariance matrix is not included. Three features of this comparison are apparent. First, when including the MHOU, the increase in PDF uncertainty in the data region is quite moderate, in agreement with
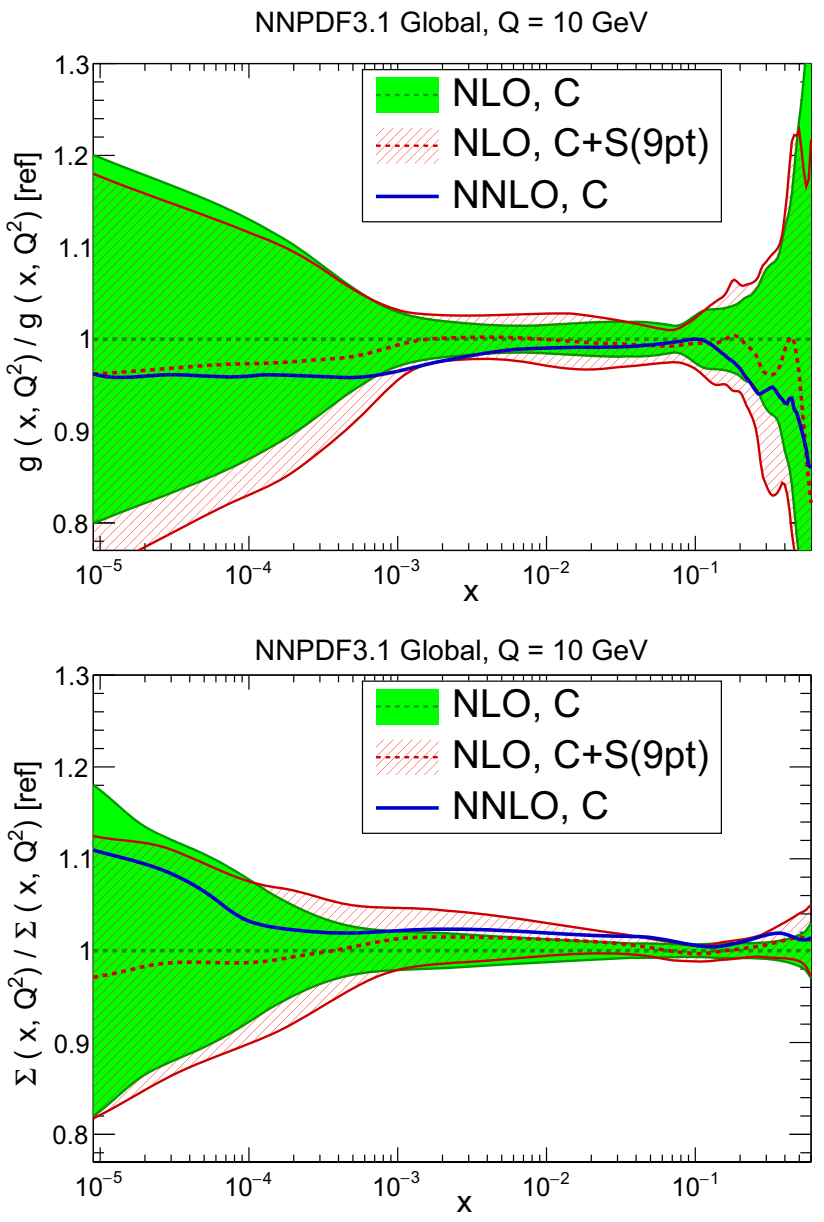

Fig. 4 The gluon and quark singlet PDFs from the NNPDF3.1 NLO fits without and with the MHOU (9-points) in the covariance matrix at $Q=10 \mathrm{GeV}$, normalized to the former. The central NNLO result is also shown

the $\phi$ values of Table 2. Second, the NLO-NNLO shift is fully compatible with the overall uncertainty. Finally, the central value is also modified by the inclusion of $S_{i j}$ in the fit, as the balance between different data sets adjusts according to their relative theoretical precision. Interestingly, the central prediction shifts towards the known NNLO result, showing that, thanks to the inclusion of the MHOU, the overall fit quality has improved.

Finally, in Fig. 5 we compare the dependence of the fit results on the specific choice of prescription for $S_{i j}$, specifically for the 3-and 9-point cases, normalized to the latter. In general the two results are consistent, but results with the 3point prescription have somewhat smaller uncertainties and, more importantly, their central value is closer to that when the MHOU is not included (see Fig. 4), so that the improved agreement between the NLO and full NNLO noted in Fig. 4 would be mostly lost if the 3-point prescription were adopted, providing further confirmation for preferring the 9-point prescription. 


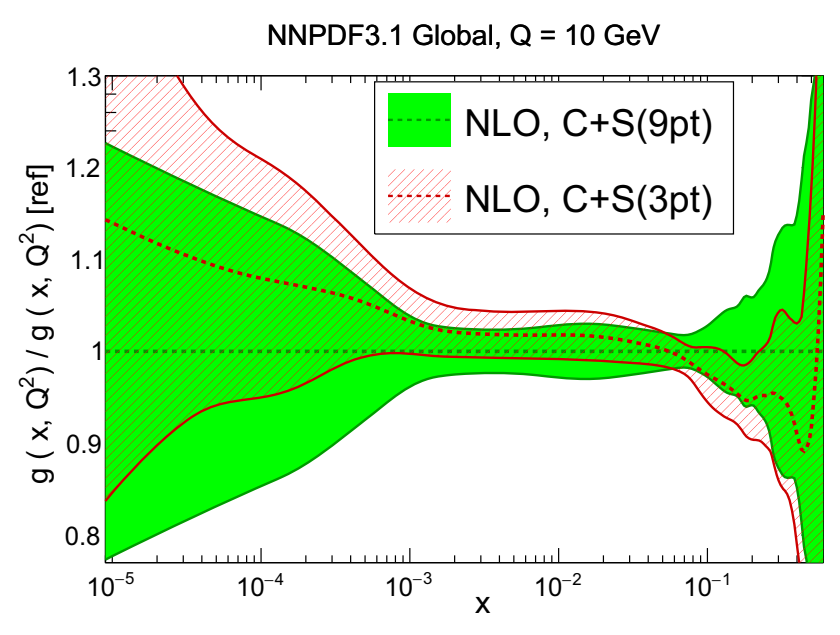

Fig. 5 Same as Fig. 4 for the gluon, comparing the 3-point and 9-point prescriptions as a ratio to the latter

It is important to understand that the meaning of PDFs and their uncertainties changes once the theory covariance matrix is included: so the error bands e.g. in Fig. 4 have a different meaning according to whether the theory covariance matrix is included. When it is included, PDF uncertainties account for data and methodological uncertainties, but also for MHOUs. Also, their central values now optimize the agreement with data based on a $\chi^{2}$ which includes MHOUs.

The usage of these PDFs is accordingly different. Firstly, they should be combined with hard cross-sections which also include MHOU. The MHOU on the prediction and the PDF uncertainty (now also including MHOUs) should be combined in the standard way (i.e. in quadrature), since with a universal PDF it is not possible to keep track of the correlations (which surely exist) between MHOU in processes used for PDF determination, and the MHOU in the prediction itself. This neglected correlation is likely to be a small effect in most situations [12], and it leads to a conservative uncertainty estimate. Second, it is important to keep in mind that MHOUs in the theory prediction must be included in the computation of the $\chi^{2}$ when assessing the agreement of these PDFs with new data, since, as we have seen, their central value is shifted as a consequence of the inclusion of the MHOUs.

In summary, we have presented the first global PDF analysis that accounts for the MHOU associated with the fixed order QCD perturbative calculations used in the fit. The inclusion of the MHOU shifts central values by an amount that is not negligible on the scale of the PDF uncertainty, moving the NLO result towards the NNLO result. PDF uncertainties increase moderately, because of the improvement of fit quality due to the rebalancing of datasets according to their theoretical precision. For this to be effective, the correlations in $S_{i j}$ play a crucial role. These correlations are rather more extensive than those related to experimental system- atics, since all different measurements of the same process are correlated through their common MHO corrections, and different processes are correlated through $\mathrm{MHO}$ corrections to perturbative evolution. A more accurate treatment of these correlations (especially those related to perturbative evolution) will be the subject of future studies.

Our results pave the way towards a fully consistent treatment of MHOU for precision LHC phenomenology. The NLO results presented here will be upgraded to NNLO, facilitated by tools such as the APPLfast grid interface to the NNLOJET program [19]. We thus anticipate that the upcoming NNPDF4.0 PDF set will be able to fully account for MHOU both at NLO and NNLO, as well as other sources of theory uncertainty, such as those related to nuclear corrections $[10,20]$.

Acknowledgements R. D. B. is supported by the UK Science and Technology Facility Council through Grant ST/P000630/1.S. F. is supported by the European Research Council under the European Union's Horizon 2020 research and innovation Programme (Grant agreement n.740006). T. G. is supported by The Scottish Funding Council, grant H14027. Z. K. is supported by the European Research Council Consolidator Grant "NNLOforLHC2". E. R. N. is supported by the European Commission through the Marie Skłodowska-Curie Action ParDHonS_FFs.TMDs (Grant number 752748). R. L. P. and M. W. are supported by the STFC Grant ST/R504737/1. J. R. is supported by the European Research Council Starting Grant "PDF4BSM" and by the Netherlands Organization for Scientific Research (NWO). L. R. is supported by the European Research Council Starting Grant "REINVENT" (Grant number 714788). M. U. is partially supported by the STFC Grant ST/L000385/1 and funded by the Royal Society grants DH150088 and $\mathrm{RGF} / \mathrm{EA} / 180148$. C. V. is supported by the STFC grant ST/R504671/1.

Data Availability Statement This manuscript has associated data in a data repository. [Authors' comment: Parton distributions with theory uncertainties discussed here are publicly available in LHAPDF format from the NNPDF website: http://nnpdf.mi.infn.it/nnpdf3-1th/.]

Open Access This article is distributed under the terms of the Creative Commons Attribution 4.0 International License (http://creativecomm ons.org/licenses/by/4.0/), which permits unrestricted use, distribution, and reproduction in any medium, provided you give appropriate credit to the original author(s) and the source, provide a link to the Creative Commons license, and indicate if changes were made.

Funded by SCOAP ${ }^{3}$.

\section{References}

1. D. de Florian et al., Handbook of LHC higgs cross sections: 4. Deciphering the nature of the higgs sector. https://doi.org/10. 2172/1345634, https://doi.org/10.23731/CYRM-2017-002 (2016) arXiv:1610.07922

2. M. Cepeda, et al., (2019). arXiv:1902.00134

3. J. Gao, L. Harland-Lang, J. Rojo, Phys. Rep. 742, 1 (2018). arXiv:1709.04922

4. J. Rojo et al., J. Phys. G 42, 103103 (2015). arXiv:1507.00556

5. R.D. Ball et al., Eur. Phys. J. C 77(10), 663 (2017). arXiv: 1706.00428

6. M. Cacciari, N. Houdeau, JHEP 1109, 039 (2011). arXiv:1105.5152 
7. A. David, G. Passarino, Phys. Lett. B 726, 266 (2013). arXiv:1307.1843

8. E. Bagnaschi, M. Cacciari, A. Guffanti, L. Jenniches, JHEP 02, 133 (2015). arXiv: 1409.5036

9. R. D. Ball, A. Deshpande, The proton spin, semi-inclusive processes, and measurements at a future electron ion collider, in From My Vast Repertoire ... : Guido Altarelli's Legacy, ed. By A. Levy, S. Forte, G. Ridolfi (World Scientific, Singapore, 2018). arXiv: 1801.04842

10. R.D. Ball, E.R. Nocera, R.L. Pearson, Eur. Phys. J. C 79(3), 282 (2019). arXiv: 1812.09074

11. R.L. Pearson, C. Voisey, Nucl. Part. Phys. Proc. 300-302, 24 (2018). arXiv: 1810.01996

12. R. Abdul Khalek, et al., (2019). arXiv:1906.10698

13. R.D. Ball, S. Carrazza, L. Del Debbio, S. Forte, Z. Kassabov, J. Rojo, E. Slade, M. Ubiali, Eur. Phys. J. C 78(5), 408 (2018). arXiv: 1802.03398

14. Z. Kassabov. Reportengine: a framework for declarative data analysis. https://doi.org/10.5281/zenodo.2571601 (2019)

15. V. Bertone, S. Carrazza, J. Rojo, Comput. Phys. Commun. 185, 1647 (2014). arXiv:1310.1394
16. V. Bertone, S. Carrazza, N.P. Hartland, Comput. Phys. Commun. 212, 205 (2017). arXiv: 1605.02070

17. T. Carli et al., Eur. Phys. J. C 66, 503 (2010). arXiv:0911.2985

18. R.D. Ball et al., JHEP 04, 040 (2015). arXiv: 1410.8849

19. T. Gehrmann, et al., PoS RADCOR2017, 074 (2018). arXiv: 1801.06415

20. R. Abdul Khalek, J.J. Ethier, J. Rojo, Eur. Phys. J. C 79, 471 (2019). arXiv:1904.00018 